\title{
Cloning, heterologous expression, and sequencing of a novel proline iminopeptidase gene, pepl, from Lactobacillus delbrueckii subsp. lactis DSM 7290
}

\author{
Jürgen R. Klein, Ulrike Schmidt and Roland Plapp
}

Author for correspondence: Jürgen R. Klein. Tel: +49631205 2179. Fax: +496312052998.

Fachbereich Biologie, Abteilung Mikrobiologie, Universität Kaiserslautern, Postfach 3049, 67653 Kaiserlautern, Germany

\begin{abstract}
The gene for proline iminopeptidase from Lactobacillus delbrueckii subsp. lactis DSM 7290 coding for an enzyme that hydrolyses the synthetic substrate L-prolyl- $\beta$-naphthylamide (Pro- $\beta$ NA) was cloned in Escherichia coli. An enzymic plate assay was used to screen for positive clones. The gene, designated pepl, was subcloned into vector pUC18 and sequenced. The nucleotide sequence revealed an 882 bp open reading frame encoding 294 amino acids, coding for an enzyme with a calculated molecular mass of $32883 \mathrm{Da}$. By cloning under control of the lac promoter the peptidase was highly expressed. Sequence analysis showed that pepl is of a new sequence type, distinct from all peptidases so far sequenced. Amino acid homology to the active site of a Pseudomonas putida esterase and inhibitor studies of the enzyme imply involvement of a serine residue in catalysis.
\end{abstract}

Keywords: iminopeptidase, serine protease, Lactobacillus delbrueckii subsp. lactis, nucleotide sequence analysis

\section{INTRODUCTION}

Lactobacilli growing in milk need to possess an efficient enzymic system to utilize the abundant nutrients present. Using a coordinated combination of proteinases, peptidases, and amino acid and peptide transport systems, they are able to use milk protein, especially casein, as a source of amino acids essential for growth. The proteolytic system of lactobacilli used as starter cultures in the industrial dairy fermentations is an important factor influencing dairy product quality (e.g. organoleptic properties, or taste, and texture). This has resulted in increased fundamental research concerning the genes and corresponding enzymes involved, which may contribute to the improvement of industrial strains. Additionally the genes of individual proteinases or peptidases may serve as food-grade markers in plasmids suitable for genetic engineering.

The large peptides generated by proteinases during casein

Abbreviations: 3,4-DCl, 3,4-dichloroisocoumarin; E-64; L-trans-epoxysuccinylleucylamide(4-guanidino)-butane; $p$-NA, $p$-nitroanilide; Pro- $\beta$ NA, Lprolyl- $\beta$-naphthylamide.

The GenBank/EMBLDDBJ accession numbers for the novel nucleotide sequence data reported in this paper are $Z 26948$ and $Z 26951$. degradation are further hydrolysed into smaller peptides and amino acids by the action of peptidases. The catalytic activity of the proline iminopeptidase (EC 3 .4.11.5) is to cleave $\mathrm{N}$-terminal proline residues from peptides. This bond is less susceptible to the action of aminopeptidases of broad specificity because proline residues confer structural constraints on the peptide (Yaron \& Naider, 1993). Casein has an extraordinarily high content of proline residues $(16.7 \%$ for $\beta$-casein; Casey \& Meyer, $1985)$ and the iminopeptidase is able to remove the blocking proline residues, thus making the peptides again accessible for other enzymes. Proline iminopeptidase activity has been reported for a variety of organisms, but as far as we know only a Bacillus gene has been cloned and sequenced (Kitazono et al., 1992). This paper deals with the cloning, sequencing and expression of the proline iminopeptidase from Lactobacillus delbrueckii subsp. lactis DSM 7290 in Escherichia coli.

\section{METHODS}

Bacterial strains, plasmids, and growth conditions. Escherichia coli K12 strain ER1562 (Raleigh et al., 1988) was used for molecular cloning and strain CM89 (Miller \& Schwartz, 1978) for preparation of cell extracts. The low-copy-number vector pLG339 (Stoker et al., 1982) was from N. Stoker and pUC18 
(Yanisch-Perron et al., 1985) was obtained from Pharmacia. E. coli was grown at $37^{\circ} \mathrm{C}$ in Luria-Bertani (LB) medium (Sambrook et al., 1989) at $37^{\circ} \mathrm{C}$. Ampicillin or kanamvcin were added to concentrations of $200 \mu \mathrm{g} \mathrm{ml}^{-1}$ and $40 \mu \mathrm{g} \mathrm{ml}^{-1}$, respectively.

Transformations. E. coli was transformed by electroporation using a Bio-Rad Gene Pulser as described by Dower et al. (1988).

Recombinant DNA techniques. Restriction enzymes and other nucleic-acid-modifying enzymes were used as recommended by the manufacturers. Isolation of plasmid DNA from E. coli was performed as described by Sambrook et al. (1989).

Isolation of chromosomal DNA from $L$. delbrueckii subsp. lactis DSM 7290 and preparation of a plasmid library in the low copy number vector pLG339 (Stoker et al., 1982) was performed as described previously (Klein et al., 1993).

Molecular cloning of the $L$. delbrueckii subsp. lactis pepl gene. A gene bank of size-fractionated partial Sau3A fragments of total $L$. delbrueckii subsp. lactis DNA constructed with vector pLG339 (Stoker et al., 1982) was used to transform E. coli strain ER1562 as described previously (Klein et al., 1993). Colonies with proline iminopeptidase activity were identified by a plate staining method originally described by Miller \& Mackinnon (1974). If the chromogenic substrate Pro- $\beta \mathrm{NA}$ is cleaved, the reaction of the $\beta$-naphthylamine with fast garnet GBC (Sigma) can be monitored by the formation of a red, non-diffusible azo dye. During subcloning the transformants, selected on ampicillin- or kanamycin-containing LB-plates, were screened for those showing proline iminopeptidase enzyme activity with Pro- $\beta$ NA as a substrate

DNA sequence analysis. DNA sequencing of the chromosornal insert of pJK505 was carried out with a pair of universal sequencing primers adjacent to the multiple cloning site of pUC18. Synthetic oligonucleotide primers, deduced from the investigated sequence, were synthesized (Applied Biosystems model 392), and allowed direct sequencing of double-stranded plasmid DNA purified by NUCLEOBOND AX100 columns (Machery-Nagel). The DNA sequence of each strand was determined using the T7 DNA polymerase sequencing kit (Pharmacia), which is based on the dideoxynucleotide chaintermination method (Sanger et al., 1977), in the presence of $\left[{ }^{35} \mathrm{~S}\right] \mathrm{dATP} \alpha \mathrm{S}$ (Amersham). For computer-assisted sequence analysis the Microgenie (Beckman), PC-Gene (IntelliGenetics), and HUSAR (GENIUSnet) software were used.

Preparation of cell extracts. Cell pellets of E. coli from a $50 \mathrm{ml}$ overnight culture were washed with $50 \mathrm{mM}$ Tris $/ \mathrm{HCl} \mathrm{pH} 8.0$, pelleted by centrifugation and resuspended in $500 \mu \mathrm{l}$ of the same buffer. The bacteria were sonicated on ice (Bandelin sonifier; Sonopuls HD60) until more than $90 \%$ of the cells were broken. Cell debris was removed by centrifugation at $51000 \mathrm{~g}$ and $4{ }^{\circ} \mathrm{C}$ for $60 \mathrm{~min}$ in a Hereus Biofuge RS28.

The supernatant of the preparation contained approximately $50 \mathrm{mg}$ protein $\mathrm{ml}^{-1}$ as determined by the Lowry method and was used for enzyme assays and SDS-PAGE (Laemmli, 1970). The content of PepI protein in cell extracts was determined videodensitometrically (Cybertech CS1, Image Documentation System) with Coomassie-stained SDS-gels, calibrated with bovine serum albumin standards.

Enzyme assay and effects of various chemical reagents. For characterization of the enzyme, $p$-nitroanilide $(p-\mathrm{NA})$ substrates (Bachem) were dissolved in water and added to the reaction mixture $[10 \mathrm{mM}$ Tris $/ \mathrm{HCl}, \mathrm{pH} 8 \cdot 0$, and varying amounts of cell extracts $(10-2000 \mathrm{ng})]$ to a concentration of $1 \mathrm{mM}$ in a volume of $250 \mu \mathrm{l}$. Release of $p$-nitroaniline was measured after a $10 \mathrm{~min}$ incubation at $37^{\circ} \mathrm{C}$ at $405 \mathrm{~mm}$ in a LKB Ultrospec Plus spectrophotometer.

To study the mechanism of enzyme action, the inhibitors 3,4dichloroisocoumarin (3,4-DCI) at a concentration of $0.1 \mathrm{mM}$, pepstatin $\mathrm{A}$ at $1 \mu \mathrm{g} \mathrm{ml}^{-1}$, L-trans-epoxysuccinylleucylamide(4guanidino)-butane (E-64) at $0.1 \mathrm{mM}$, and 1,10-phenanthroline or EDTA at $1 \mathrm{mM}$ were added to the extracts and incubated for $30 \mathrm{~min}$ at $37^{\circ} \mathrm{C}$. The substrate Pro- $p$-NA was added and activity was measured spectrophotometrically by the release of $p$ nitroaniline.

\section{RESULTS AND DISCUSSION}

\section{Cloning of the L. delbrueckii subsp. lactis DSM7290 pepl gene}

E. coli ER 1562 was transformed with the plasmid library of DSM 7290 chromosomal DNA in pLG339 and the pepI gene could be isolated from colonies showing peptolytic activity on the substrate Pro- $\beta$ NA. In the plate assay this substrate was not cleaved by the E. coli host strain, nor was it cleaved by $E$. coli harbouring the recently cloned genes for X-prolyl-dipeptidyl aminopeptidase (MeyerBarton et al., 1993) and aminopeptidase $\mathrm{N}$ (Klein et al., 1993) from $L$. delbrueckii subsp. lactis DSM 7290. From approximately 6500 transformants screened, four Pro$\beta$ NA-cleaving colonies could be detected. Restriction

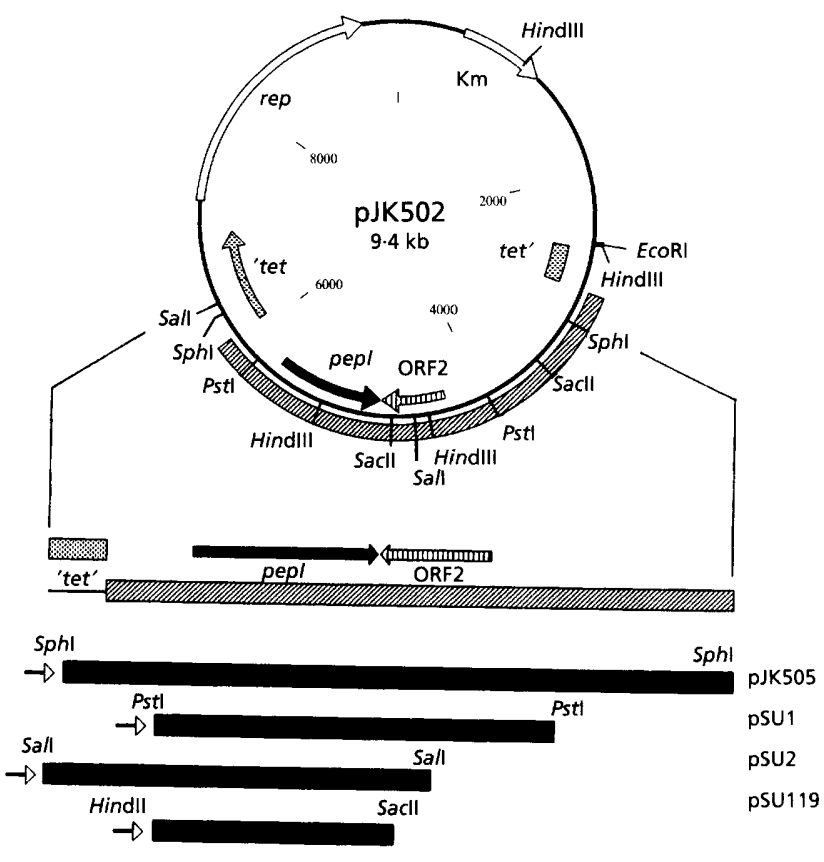

Fig. 1. Genetic map of $\mathrm{pJK} 502$. The $3 \cdot 2 \mathrm{~kb}$ insert of chromosomal DNA is indicated as a hatched segment, with the pepl gene located along the black arrow. The linear expansion of the insert illustrates the subcloning steps. The different restriction fragments used for subcloning into vector pUC18, all spanning the pep/ gene, are indicated as shaded bars. 
analysis revealed that all plasmids isolated had inserts ranging in size from 3.2 to $8.0 \mathrm{~kb}$, with a conserved identical core region. Plasmid pJK502, with the smallest insert size of $3 \cdot 2 \mathrm{~kb}$, was subjected to further analysis (Fig. 1).

\section{Subcloning and nucleotide sequence analysis}

Subcloning of a $3 \cdot 2 \mathrm{~kb} S p h I$ fragment from pJK502 in pUC18 resulted in plasmid pJK505 (Fig. 1) expressing the Pepl activity coded on a high-copy-number plasmid. The complete nucleotide sequence of both strands of this $S p h \mathrm{I}$ fragment was determined using synthetic oligonucleotide primers deduced from the investigated sequences. Analysis of the nucleotide sequence determined $(2958 \mathrm{bp}, 51 \cdot 4$ $\mathrm{mol} \% \mathrm{C}+\mathrm{G}$ ) uncovered the putative open reading frame for pepI (Fig. 2) extending from an ATG codon at nucleotide 410 to a TAA stop codon at position 1292 , which is sufficient to encode a protein of 294 amino acids corresponding to a protein with a molecular mass of $32883 \mathrm{Da}$. A second open reading frame (ORF2) located on the opposite DNA strand, ranging from ATG at position 1812 to the TAG stop codon at position 1291, is capable of coding a protein of 173 amino acids with a molecular mass of $19822 \mathrm{Da}$.

\section{Overexpression of Pepl protein in E. coli}

Crude cell extracts of E. coli CM89 harbouring plasmids pJK502 or pJK505, both coding for pepI, were subjected to SDS-PAGE (Fig. 3). A $34 \mathrm{kDa}$ protein could be detected; this value corresponds well with the molecular mass of the enzyme predicted from nucleotide sequence analysis.

The subcloning experiments, which were performed in order to minimize the pepI coding region, are outlined in Fig. 1. Cloning of the $3 \cdot 2 \mathrm{~kb} S p h \mathrm{I}$ fragment from the lowcopy-number plasmid pJK502 into high-copy-number vector $\mathrm{pUC18}$, resulting in $\mathrm{pJK} 505$, increased gene dosage, but this had only a minor effect on protein overexpression. The knowledge of the complete pJK505 nucleotide sequence allowed cloning of defined DNA fragments. The Pst I fragment from pJK505 was subcloned into pUC18, both possible insert orientations were obtained and both constructs expressed PepI protein, thus indicating that the pepI promoter sequence is functional in E. coli. But only the plasmid (pSU1) with the lac promoter upstream of pepI showed a drastic increase of PepI expression (Fig. 3). Plasmid pSU2, with pepI on a $1.8 \mathrm{~kb}$ $S_{a l l}$ fragment, has a spacing region of $1 \mathrm{~kb}$ between the lac promoter and the structural gene and PepI is less efficiently expressed than in pSU1. We further succeeded in cloning the small pepI-expressing $1140 \mathrm{bp} \mathrm{HincII/SacII}$ fragment from pSU1 into pUC18, resulting in pSU119.

The amount of PepI protein expressed was correlated with the copy number of the vectors used, but a considerable increase could be observed after cloning under control of the lac promoter. In E. coli CM89(pSU119) the pepI product constituted about 50\% of the cytoplasmic proteins (Fig. 3). This remarkable high-level expression of the pepI product appeared not to be lethal for the E. coli host, but the high-copy-number plasmids were structurally unstable and PepI activity was lost after approximately 80 generations with antibiotic selection. In earlier experiments with pepX (encoding Xprolyl-dipeptidyl aminopeptidase; Meyer-Barton et al., 1993), we were not successful in cloning the peptidase gene into pUC18, and with $p e p N$ (aminopeptidase $\mathrm{N}$; Klein et al., 1993) subcloning into high-copy-number vectors resulted in extremely unstable plasmids. A reason for this was probably the strong overexpression in E. coli under control of the original promoter even if cloned in low-copy-number plasmids.

A protein which could be associated with ORF2 gene product $(19 \cdot 8 \mathrm{kDa})$ was not detectable by SDS-PAGE.

\section{Homologies of the $L$. delbrueckii subsp. lactis sequence}

Searching the EMBL database with the investigated nucleotide sequence resulted in no significant homologies; only after translation of the two proposed open reading frames were sequences with homologies detected in the protein databases.

The ORF2 gene product is similar to a repressor protein of Bacillus subtilis pai1 (Honjo et al., 1990) (Fig. 4). In Bacillus this repressor is involved in reduction of extracellular and cell-associated protease levels, when present in high copy number, and it is considered to function as a transcriptional regulator. The physiological function of the lactobacillal ORF2 gene product remains to be investigated.

For pepI the most significant homology is observed in a small, but functionally important segment of a 2-hydroxy6-oxohepta-2,4-dienoate hydrolase (Menn et al., 1991), an enzyme involved in degradation of toluene, which contains the serine catalytic site (Fig. 5). Comparison of both protein sequences over their entire length results only in a non-significant homology of $23.8 \%$ (70 in 294 amino acids). This result shows that $p e p I$ is of new sequence type, distinct from prolyl-aminopeptidase, the only one having so far been cloned and sequenced being from Bacillus coagulans (Kitazono et al., 1992), also distinct from the Pro$\beta$-naphthylamidase from porcine liver (Matsushima $e t$ al., 1991), which turned out to be a carboxylesterase, and from all other aminopeptidases hitherto sequenced.

The absence of a signal peptide sequence and the hydrophilicity plot of the amino acid sequence according to Kyte \& Doolittle (1982), which does not show transmembrane domains, indicate that Pepl of $L$. delbrueckii subsp. lactis might be an intercellularly located enzyme. No secreted peptidase has yet been found in lactic acid bacteria. However, an extracellular location of peptidases is discussed since most peptides released from casein by the proteinase are too large to be taken up by the 
SaU3A

1 GATCATTGAACTCGTCAAAAACATAGCTGGTCTCAATCGTATGGAAAGAAAAGAGGTTCTTGTCCTGTAGCAAAAAAGTGATTGGCCGGGAAGTGAAGTG CTAGTAACTTGAGCAGTTTTTGTATCGACCAGAGTTAGCATACCTTTCTTTTTCTCCAAGAACAGGACATCGTTTTTTCACTAACCGGCCCTTCACTTCAC

101 CTTGATGGTCGTCGTTGGCCAGACTGGCACGTCATAGACTAGCAGGTGGGTGTCGGTATATTTGGTCGTAGTCGTAGTGGGGCCGTTCATGCCGGTCGGAA GAACTACCAGCAGCAACCGGTCTGACCGTGCAGTATCTGATCGTCCACCCACAGCCATATAACCAGCATCAGCATCACCCCGGCAAGTACGGCCAGCCTT

201 ACGTAGGAAATGATTTCCGGTGTAAAATAAAACTCTGTCMPCAgGCGCCGTCTGTCAGCTTCGTTCAAGCCATTGACGTCATACCAGTTAAAGTCTGTTT' TGCATCCTTTACTAAAGGCCACATTTTATTTTGAGACAGACGTCCGCGGCAGACAGTCGAAGCAAGTTCGGTAACTGCAGTATGGTCAATTTCAGACAAA

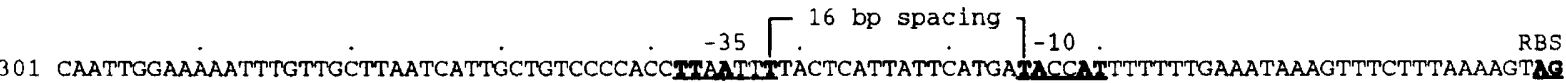
GTTAACCTTTTTAAACAACGAATTAGTAACGACAGGGGGGAATTAAAATGAGTAATAAGTACTATGGTAAAAAAACTTTATTTCAAAGAAATTTCATC

401 GMAAAAAAGATGCAAATCACAGAAAAATATCTTCCATTTGGAAATTGGCAAACCTACTGCCGGATCGTGGGCGAGGCTACTGACCGCGCCCCGCTCCTCC

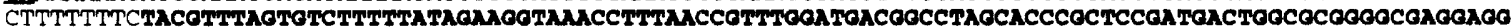
$\begin{array}{lllllllllllllllllllllllllllllll}M & Q & I & T & E & K & Y & L & P & F & G & N & W & Q & T & Y & C & R & I & V & G & E & A & T & D & R & A & P & L & L & L\end{array}$ PopI $-\cdots+\ldots-\infty$

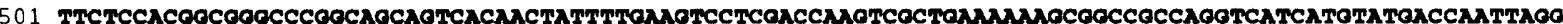
AAGAGGTGCCGCCCGGGCCGTCGTCAGTGTTGATAAAACTTCAGGAGCTGGTTCAGCGACTTTTTTCGCCGGCGGTCCAGTAGTACATACTGGTTAATCC

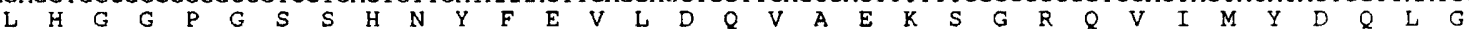

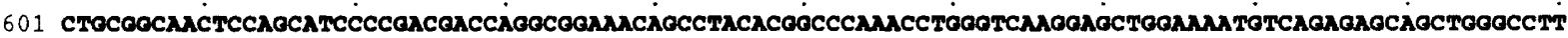
GACGCCGTTGAGGTCGTAGGGGCTGCTGGTCCGCCTTTGTCGGATGTGCCGGGTTTGGACCCAGTTCCTCGACCTTTTACAGTCTCTCGTCGACCCGGAA

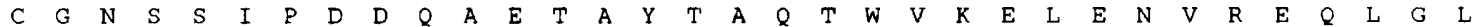

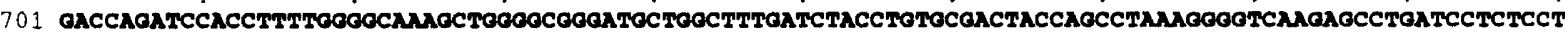
CTGGTCTAGGTGGAAAACCCCGTTTCGACCCCGCCCTACGACCGAAACTAGATGGACACGCTGATGGTCGGATTTCCCCAGTTCTCGGACTAGGAGAGGA

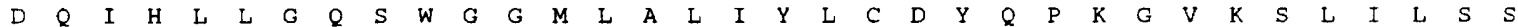

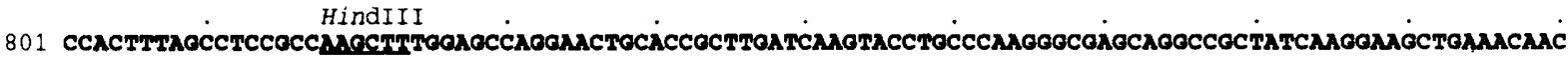
GGTGAAATCGGAGGCGGTTCGAAACCTCGGTCCTTGACGTGGCGAACTAGTTCATGGACGGGTTCCCGCTCGTCCGGCGATAGTTCCTTCGACTTTGTTG

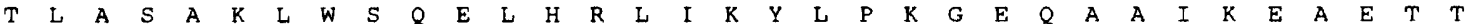

901 ToOcג ACCGTTGATGCTGAGGGGCCGGATGGTCCGCCGGTTACGGGTGAAGTACCTGGTCGTGCGGTAGTTGCAATGCGGCCTGGACGGCCTCGGTCAAAACGCG

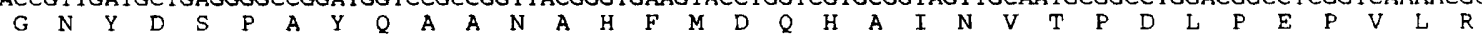

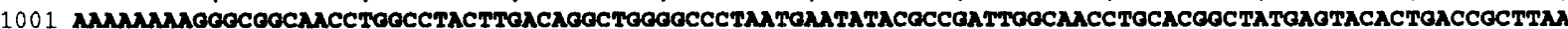
TTTTTTTTCCCGCCGTTGGACCGGATGAACTGTCCGACCCCGGGATTACTTATATGCGGCTAACCGTTGGACGTGCCGATACTCATGTGACTGGCGAATT

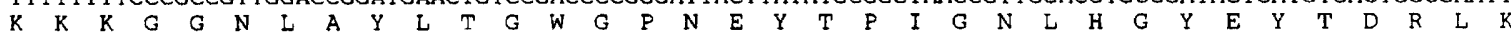

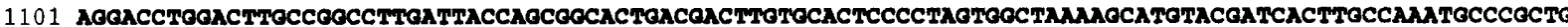
TCCTGGACCTGAACGGCCGGAACTAATGGTCGCCGTGACTGCTGAACACGTGAGGGGATCACCGATTTTCGTACATGCTAGTGAACGGTTTACGGGCGAC

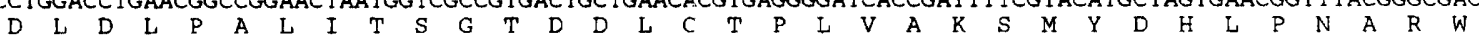

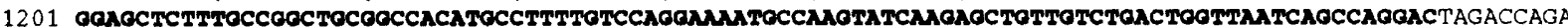

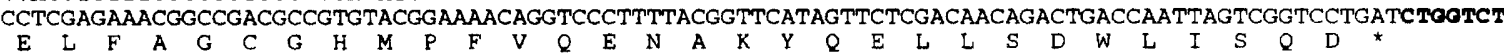

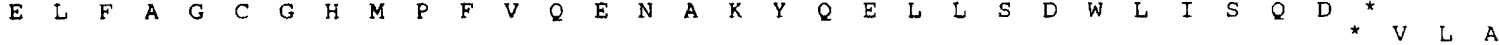

1301 GCCTTCTTTAACAAAAAATCTGTCTGACGGTCATCGCCAAGAACGAAGACATGCTCGCTAAACCGGGTCAAACCOCPOCTGGCATAGAAGTTCTTGGCGT

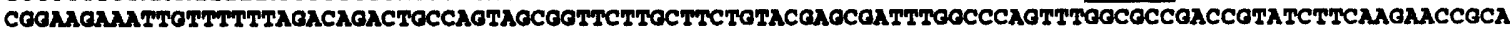

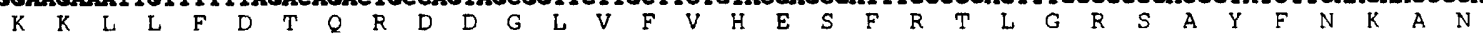

1401 TTTCGTTGTGCTCCCAAACGCCCAGCCAGAGGACAGACTTGCTCCATTCCCGGCCCTTTTCTTCCGCGAACTCAAAGAGCTGCTTGCCCAGGCCCCGGTC

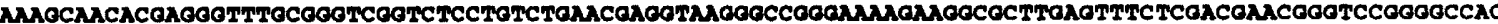

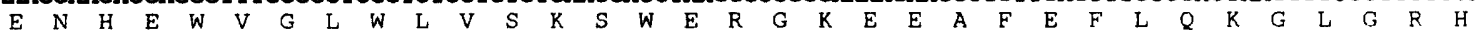

SalI

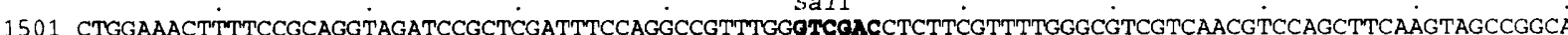

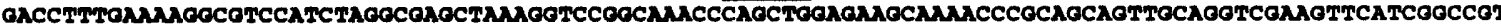

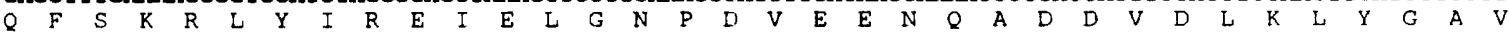
HindII I

1601 ACTTCCCCGTCCACCTTCAAGAAGTAAAAGAAGCTGCCCGGAGTTGCTATTTCTCCCCCMACHYGTCTTCATTGATGGTTTTCTCCAAAAACTTGGCCA

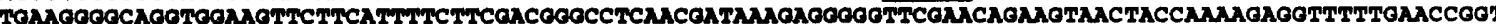

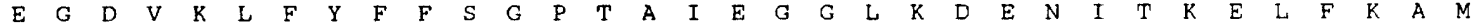

1701 TATCTTCCGGGCTGTTGTCTTTGCCAAAAGTATCCGCGAAAGTTTCCCGGCTGATGGCAATGAACTCTGGCAAATCTTTCTCACTAACTITGCACAATTTT

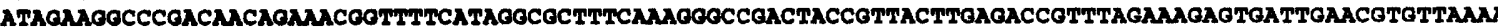

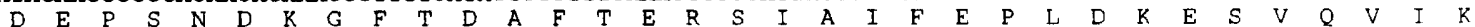

Fig. 2. For legend see facing page. 
1801 TACTCCTGTCATGCTTTGCCTTTTTCTCTTTTTCTGCTTTACAGTTTCTTAGTTAAGACCCAGGTTCTGAGCAGCATCCCTGCTCTTTCCCAGTCTTCTT ATOAのOA CגOTACGAAACGGAAAAAGAGAAAAAGACGAAATGTCAAAGAATCAATTCTGGGTCCAAGACTCGTCGTAGGGACGAGAAAGGGTCAGAAGAA $\mathrm{V}$ G T M $<\ldots$ ORF2

1901 CCCGGTAGTCGTGGCCAGCGCCCCGCATTTGCAAAAGTGTCGCCTTGCTTCAGCTGCTTGGCCATCATTTCCAGGCTGGCAAAGGCGCCTGGTCGTCATC GGGCCATCAGCACCGGTCGCGGGGCGTAAACGTTTTCACAGCGGAACGAAGTCGACGAACCGGTAGTAAAGGTCCGACCGTTTCCGCGGACCAGCAGTAG

2001 GCTGCCGGAAACAATGTAGCTGCGGCCCTGGTAGCCCTTGATCTTGTCCCAGTCCTTGATGACTGGTGACATTAAAAAGATCCCCCGGACTGACCGGCCG CGACGGCCTTTGTTACATCGACGCCGGGACCATCGGGAACTAGAACAGGGTCAGGAACTACTGACCACTGTAATTTTTCTAGGGGGCCTGACTGGCCGGC

Pst I

2101 ATTTCACTAGCCGCAACAGCTEGAOCGTTGCCCGCTTCCCCGTAGCCGGCAAAGACGATGTATTCGCTGGCCACCCGGTCATCGTTTCTAAGTTCGTCAA TAAAGTGATCGGCGTTGTCGACGTCGCAACGGGCGAAGGGCATCGGCCGTTTCTGCTACATAAGCGACCGGTGGGCCAGTAGCAAAGATTCAAGCAGTT

2201 AGGCCAGGTGGTAGCCGCTGATGGCGCTGGCTGTATCTTCTGCTTCAAAGGTGAAGGTCAGGATGCCGGCGTCGTTTAAGTAGCGGCTGAGCCAATAGGC TCCGGTCCACCATCGGCGACTACCGCGACCGACATAGAAGACGAAGTTTCCACTTCCAGTCCTACGGCCGCAGCAAATTCATCGCCGACTCGGTTATCCG

2301 CTGGTCCTTATCGCCGGTCTTAGCCAAAATTACCAAAGGGGTCATCCCTTCGATCGGGTTGACGTTCGCCCCAAATTCCCTTTAACTTGCCCTTGGCGCT GACCAGGAATAGCGGCCAGAATCGGTTT"TAATGGTTTCCCCAGTAGGGAAGCTAGCCCAACTGCAAGCGGGGTTTAAGGGAAATTGAACGGGAACCGCGA

2401 GATTAAGGTCATCTCTTT"TCCATAAAAACTCCTTACAATTTCTTACTTTACATTAAAAAACTTACAACATTCCTATTGTACTATTTTTTGCAGGAAAT CTAATTCCAGTAGAGAAAAAGGTATTTTTTGAGGAATGTTAAAGAATGAAATGTAATTTTTTTGAATGTTGTAAGGATAACATGATAAAAAACGTCCTTTA

2501 TAGGGCCGGTACTTGTAAATCGAGTTGTTGAGATTGTCCTTMACCAGCTGGTCAAAATTTTCTGCCCTTGGCCGTAATCGCCOCOQCAATTTGATCATTG ATCCCGGCCATGAACATTTAGCTCAACAACTCTAACAGGAACTGGTCGACCAGTTTTTAAAGACGGGAACCGGCATTAGCGGCGCCGTTAAACTAGTAAC

2601 GCTTCCGTCAAAGACCCGGCGGTTGCTGGTTCGCTTTCCGCTGAACTCATGGCCACGTTGTAGCTGTTGÄTCCCCTGCTCGTCAAAGCGGCCGTAGTCTT CGAAGGCAGTT'TCTGGGCCGCCAACGACCAAGCGAAAGGCGACTTGAGTACCGGTGCAACATCGACAACTAGGGGACGAGCAGTTTCGCCGGCATCAGAA

2701 CTACGGCGTCACGGACGGCGGTGAAGCCGCAGCCTTCTCCंCTTTAAGGGAATTTCAACGCCGGTTCCTTTGGAGACAAAGAGCTCATCATAATTTTTTAGC GATGCCGCAGTGCCTGCCGCCACTTCGGCGTCGGAAGAGGGAAATTCCCTTAAAGT"TGCGGCCAAGGAAACCTCTGTTTCTCGAGTAGTATTAAAAATCG

2801 TGGATAGTAGACAAAGCACTTTTCGTTAAAGCCCTGGTCGTAGTCTTCATCTCTGTCCACGATCGTGGACCCGTCAAGGCTGGCCTTCTTGCCCACCAGC ACCTATCATCTGTTTCGTGAAAAGCAATTTCGGGACCAGCATCAGAAGTAGAGACAGGTGCTAGCACCTGGGCAGTTCCGACCGGAAGAACGGGTGGTCG

2901 ATGGTTGTACAGTTGTCGCGCATTTTTCTTCCTTCTTTCTATTCGCTGACCTECAMPC 2958 TACCAACATGTCAACAGCGCGTAAAAAGAAGGAAGAAAGATAAGCGACTGGACGTACG

Fig. 2. Nucleotide sequence of the chromosomal insert of pJK505. The upper strand codes for pep/ (nt 410-1291) and the lower strand for a gene (nt 1812-1294) with high homology to the pai1 repressor of $B$. subtilis. The deduced amino acid sequences are given below the nucleotide sequence. Five base pairs upstream of the putative pepl ATG start codon is a ribosome-binding site with a tetranucleotide stretch (AGGA) complementary to the E. coli 16S rRNA (Shine \& Dalgarno, 1974); sequences that resemble promoter - 10 and -35 elements (Van de Guchte et al., 1992) are underlined (conserved nucleotides in bold type). An inverted repeat (overlined at bp 1396 to 1422) with a $\Delta G$ value of $-18 \mathrm{kcal} \mathrm{mol}^{-1}$ $\left(-75.3 \mathrm{~kJ} \mathrm{~mol}^{-1}\right)$ might function as transcription terminator. Some pertinent restriction enzyme sites are also indicated.

cell (Tan et al., 1993). Possibly a yet unknown mechanism might be responsible for translocation of peptidases to the cell surface.

\section{Partial characterization of the overproduced peptidase I}

Since PepI protein was highly overproduced in E. coli harbouring the gene coded on plasmid pSU119, cell extracts were prepared from strain CM89(pSU119). This strain, lacking the peptidase genes $p e p A, p e p B$, $p e p D$, $p e p N$ and $p e p Q$, was chosen to exclude as much background peptidase activity as possible.

To determine the mechanism of enzyme action, we treated cell extracts of CM89(pSU119) with various protease inhibitors. 3,4-DCI $(0 \cdot 1 \mathrm{mM})$ inhibited the enzyme activity to a final relative activity of $8.9 \%$, whereas pepstatin A (inhibition to $89 \%$ ), E-64 (inhibition to $86.5 \%$ ), 1,10phenanthroline or EDTA (inhibition to $84 \%$ ) had no significant effect on enzyme activity. These results suggest that the enzyme is classifiable as serine protease, which is consistent with its homology to the $P$. putida serine esterase active site (Fig. 5), implying that PepI has a catalytic centre with a serine residue.

To determine the substrate specificity of PepI a variety of $p$-NA substrates (Pro-, Ala-, Arg-, Asp-, Glu, Gly-, His-, Leu-, Lys-, Phe-, Ala-Pro-, Gly-Ala-, Gly-Phe-, Gly-Pro-, Gly-Trp-p-NA) were incubated with cytoplasmic extracts of CM89(pSU119), but among these only Pro-p-NA was effectively cleaved. This rapid test is only an indication of enzyme specificity, valuable if compared with the specificities of other cloned peptidases, and of course a more detailed characterization will be performed with purified enzyme. The purification and characterization of PepI will be described in the near future, as well as its subcellular location in Lactobacillus and determination of the $\mathrm{N}$-terminal sequence, in order to confirm the coding region of the nucleotide sequence. 


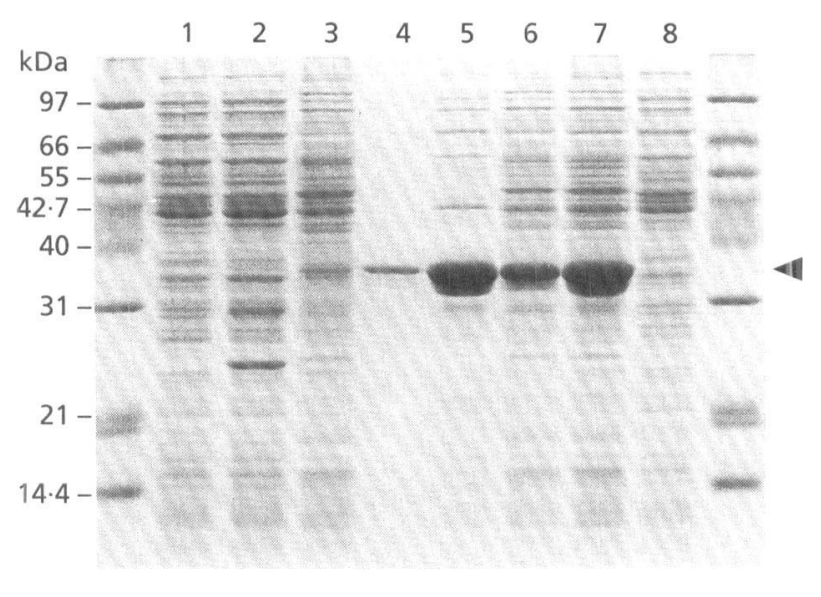

Fig. 3. Expression of Pepl protein in E. coli strain CM89. Separation of crude cell extracts by SDS-PAGE $(12 \%$, w/v, acrylamide). Proteins were visualized by Coomassie staining. The gel contained molecular mass marker proteins (both flanking lanes) and cell extracts of $E$. coli CM89(pLG339) (lane 1), CM89(pJK502) (lane 2), CM89(pJK505) (lane 3), CM89(pSU1) (lanes 4 and $5 ; 1 / 10$ of cell extract in lane 4), CM89(pSU2) (lane 6), CM89(pSU119) (lane 7) and CM89(pUC18) (lane 8). The position of Pepl is marked by an arrowhead.
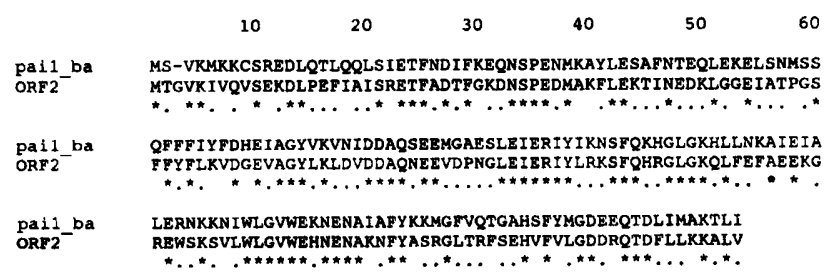

Fig. 4. Alignment resulting from CLUSTAL analysis of the ORF2 gene product (173 amino acids) as predicted from pJK505 nucleotide sequence analysis, with the $B$. subtilis pai1 repressor protein (172 amino acids), the translation product of EMBL entry M36471. Values of $45 \%$ identity and $84 \%$ similarity were calculated. Identical (*) and similar (.) amino acids are marked.

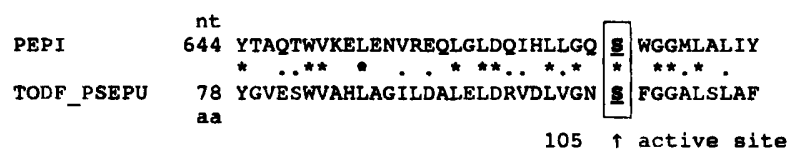

Fig. 5. Homology of the pep/ gene product with 2-hydroxy-6oxohepta-2,4-dienoate hydrolase of $P$. putida (Swissprot database entry: TODF_PSEPU) resulting from BLASTX analysis. The Pepl amino acid sequence starting at nucleotide 644 is numbered as in Fig. 2. Identities, 14/37 (37\%); positives, 23/37 (62\%).

\section{ADDENDUM}

After the completion of this work and acceptance of our manuscript, we became aware that cloning and sequencing, as well as purification and characterization, of a proline iminopeptidase from a closely related subspecies, I actobacillus delbrueckii subsp. bulgaricus, was about to be published (Atlan et al., 1994; Gilbert et al., 1994). Comparison of the sequences revealed a very high homology not only of the deduced PepI amino acid sequence (identities: 287 of 295 amino acids) but also for the up- and downstream regions of the reported nucleotide sequence. As could be expected from this, the enzymic properties of the enzymes are very similar (manuscript in preparation).

\section{ACKNOWLEDGEMENTS}

This work was supported by the Bundesministerium für Forschung und Technologie grant $0319281 \mathrm{~B}$. The authors are responsible for the content of this publication. We acknowledge the excellent and friendly assistance of Ulrike Klein and Bernd Winkelman.

\section{REFERENCES}

Atlan, D., Gilbert, C., Blanc, B. \& Portalier, R. (1994). Cloning, sequencing and characterization of the pepIP gene encoding a proline iminopeptidase from Lactobacillus delbrueckii subsp. bulgaricus CNRZ 397. Microbiology 140, 527-535.

Casey, M. G. \& Meyer, J. (1985). Presence of X-prolyl-dipeptidylpeptidase in lactic acid bacteria. J Dairy Sci 58, 3212-3215.

Dower, W. J., Miller, J. F. \& Ragsdale, C. W. (1988). High efficiency transformation of E. coli by high voltage electroporation. Nucleic Acids Res 16, 2127-2145.

Gilbert, C., Atlan, D., Blanc, B. \& Portalier, R. (1994). Proline iminopeptidase from Lactobacillus delbrueckii subsp. bulgaricus CNRZ 397: purification and characterization. Microbiology 140, 537-542.

Honjo, M., Nakayama, A., Fukazawa, K., Kawamura, K., Ando, K., Hori, M. \& Furutani, Y. (1990). A novel Bacillus subtilis gene involved in negative control of sporulation and degradative-enzyme production. J Bacteriol 172, 1783-1790.

Kitazono, A., Yoshimoto, T. \& Tsuru, D. (1992). Cloning, sequencing, and high expression of the proline iminopeptidase gene from Bacillus coagulans. J Bacteriol 174, 7919- 7925.

Klein, J. R., Klein, U., Schad, M. \& Plapp, R. (1993). Cloning, DNA sequence analysis and partial characterization of $p e p N$, a lys-aminopeptidase from Lactobacillus delbrïckii ssp. lactis DSM7290. Eur J Biochem 217, 105-114.

Kyte, J. \& Doolittle, R. F. (1982). A simple method for displaying the hydropathic character of a protein. J Mol Biol 157, 105-132.

Laemmli, U. K. (1970). Cleavage of structural proteins during the assembly of the head of bacteriophage T4. Nature 227, 680-685.

Matsushima, M., Inoue, H., Ichinose, M., Tsukada, S., Miki, K., Kuurokawa, T., Takahashi, T. \& Takahashi, K. (1991). The nucleotide and deduced amino acid sequences of porcine liver proline- $\beta$-naphthylaminidase. FEBS Lett 293, 3741.

Menn, F. M., Zylstra, G. J. \& Gibson, D. T. (1991). Location and sequence of the todF gene encoding 2-hydroxy-6-oxohepta-2,4dienoate hydrolase in Pseudomonas putida F1. Gene 104, 91-94.

Meyer-Barton, E. C., Klein, J. R., Imam, M. \& Plapp, R. (1993). Cloning and sequence analysis of the X-prolyl-dipeptidylaminopeptidase gene ( $p$ e $X)$ from Lactobacillus delbrück.ii spp. lactis DSM 7290. Appl Microbiol Biotechnol 40, 82-89.

Miller, C. G. \& Mackinnon, K. (1974). Peptidase mutants of Salmonella typhimurium. J Bacteriol 120, 355-363.

Miller, C. G. \& Schwartz, G. (1978). Peptidase deficient mutants of Escherichia coli. J Bacteriol 135, 603 611.

Raleigh, E. A., Murray, N. E., Revel, H., Blumenthal, R. M., 
Westawy, D., Reith, A. D., Rigby, P. W. J., Elhai, J. \& Hanahan, D. (1988). McrA and $\mathrm{McrB}$ restriction phenotypes of some E. coli strains and implication for gene cloning. Nucleic Acids Res 16, $1563-1575$.

Sambrook, J., Fritsch, E. F. \& Maniatis, T. (1989). Molecular Cloning: a Laboratory Manual, 2nd edn. Cold Spring Harbor, NY: Cold Spring Harbor Laboratory.

Sanger, F., Nicklen, S. \& Coulson, A. R. (1977). DNA sequencing with chain-terminating inhibitors. Proc Natl Acad Sci US A 74, 5463-5467.

Shine, J. \& Dalgarno, L. (1974). The $3^{\prime}$-terminal sequence of Escherichia coli $16 \mathrm{~S}$ ribosomal RNA: complementarity to nonsense triplets and ribosome binding sites. Proc Natl Acad Sci US A 71, 1342-1346.

Stoker, N. G., Fairweather, N. F. \& Spratt, B. G. (1982). Versatile low copy number plasmid vectors for cloning in Escherichia coli. Gene 18, 335-341.

Tan, P. S. T., Poolman, B. \& Konings, W. N. (1993). Proteolytic enzymes of Lactococcus lactis. J Dairy Res 60, 269-286.

Van de Guchte, M., Kok, J. \& Venema, G. (1992). Gene expression in Lactococcus lactis. FEMS Microbiol Rev 88, 73-92.

Yanisch-Perron, C., Vieira, J. \& Messing, J. (1985). Improved M13 phage cloning vectors and host strains: nucleotide sequence of the M13mp18 and pUC19 vectors. Gene 33, 103-119.

Yaron, A. \& Naider, F. (1993). Proline-dependent structural and biological properties of proteins. Crit Rev Biochem Mol Biol 28, 31-81.

Received 15 October 1993; accepted 24 November 1993. 